\title{
G-Series Nerve Agent GE
}

National Cancer Institute

\section{Source}

National Cancer Institute. G-Series Nerve Agent GE. NCI Thesaurus. Code C161529.

A structural analog of the nerve agent sarin, a rapid-acting non-persistent

org anophosphorus ester that is a clear, colorless and odorless liquid and is absorbed into the body by inhalation, ingestion, skin contact, or eye contact, and causes excessive cholinergic stimulation by producing potent and irreversible inhibition of cholinesterase. 